\title{
A SIMULACÃO VIRTUAL COMO FERRAMENTA PARA O DESENVOLVIMENTO DE PRODUTOS: UMA REVISÃO
}

\author{
The virtual simulation as a tool for developing products: a review
}

ROSA, Valéria llsa

UFRGS/FURB

valeriadesigner2009@hotmail.com

SILVA, Régio Pierre da

UFRGS/FURB

regio@ufrgs.br

AYMONE, José Luís Farinatti

UFRGS/FURB

aymone@ufrgs.br

\section{Resumo}

Estudo descritivo na modalidade de revisão sistemática em bases de dados, com os descritores, Tecnologia Assistiva e Simulação Virtual. Os dados foram caracterizados pela frequência em cada área de conhecimento. Assim, buscou-se nas bases de dados eletrônicas BDENF, LILACS, MEDLINE, SCIELO e SCIENCEDIRECT a Tecnologia Assistiva e a Simulação Virtual como temática nas produções de Design, entre janeiro de 2009 a junho de 2014. Das publicações, a Tecnologia Assistiva e a Simulação Virtual foram objeto em $2,74 \%$ do total dos artigos.

Palavras-chave: Revisão sistemática. Tecnologia assistiva. Simulação virtual. Desenvolvimento de produtos.

\section{Abstract}

This is a descriptive study in the form of a systematic review of databases, including descriptors, Assistive Technology and Virtual Simulation. Data was categorized by frequency in each area of expertise. Thus, we searched for Assistive Technology and Virtual Simulation (as theme in design production) in electronic databases BDENF, LILACS, MEDLINE, and SCIELO SCIENCEDIRECT, from January 2009 to June 2014. Assistive Technology and Virtual Simulation were subject to $2.74 \%$ of all articles found.

Keywords: Systematic review. Assistive technology. Virtual simulation. Product development. 


\section{INTRODUCÃO}

A Tecnologia Assistiva como enfoque de estudos científicos tem sido abordada em várias áreas de conhecimento. Destacam-se a aplicabilidade do conceito na Engenharia, Arquitetura, Medicina, Fisioterapia, Design, entre outras. Já a Simulação Virtual que pode ser caracterizada como temática tanto recente quanto o termo Tecnologia Assistiva, tem sido abordada muito mais na área da Ciência da Computação.

Os objetivos da Tecnologia Assistiva segundo Sartoretto e Bersch (2014) são proporcionar à pessoa com deficiência maior independência, qualidade de vida e inclusão social, através da ampliação de sua comunicação, mobilidade, controle de seu ambiente, habilidades de seu aprendizado, trabalho e integração com a família, amigos e sociedade.

Sendo assim, refletir sobre a Tecnologia Assistiva torna-se um desafio para designers e os mais diversos profissionais envolvidos com a realidade das pessoas com deficiência. $O$ que os estudos mais recentes revelaram sobre o tema é que a Tecnologia Assistiva é também definida como "[...] uma ampla gama de equipamentos, serviços, estratégias e práticas concebidas e aplicadas para minorar os problemas encontrados pelos indivíduos com deficiências" (COOK; HUSSEY, 1995), o que evidencia a importância de se discutir sobre o tema e a devida preocupação em desenvolver soluções baseadas em pesquisas, gerando publicações e consequentemente, produtos que possam atender as mais variadas necessidades do público em questão.

Diante da importância e escassa produção científica de ambas as temáticas, principalmente na literatura de design, esta discussão torna-se relevante, pois seus conceitos nas reflexões sobre o processo de desenvolvimento de produtos apresentam-se inovadores e atuais.

Assim, o presente artigo intenta inserir os enfoques da Tecnologia Assistiva e da Simulação Virtual como elementos de contribuição para os estudos e intervenções na área de Ciências Sociais Aplicadas, mais especificamente, do Design.

\section{METODOLOGIA}

Torna-se relevante destacar que nas pesquisas bibliográfica e documental foram obtidas informações e dados a cerca de Tecnologia Assistiva e alguns princípios sobre a Simulação Virtual.

Assim, esta pesquisa pode ser caracterizada, do ponto de vista da sua natureza, como uma pesquisa básica que Envolve verdades e interesses 
universais, procurando gerar conhecimentos novos úteis para o avanço da ciência, sem aplicação prática prevista . (PRODANOV; FREITAS, 2013, p. 126). Além disso, o presente estudo caracteriza-se quanto aos seus objetivos, como uma pesquisa exploratória que é, na visão de Triviños (2006), fundamental tanto para a pesquisa experimental quanto para a pesquisa descritiva, tendo em vista que a exploração visa à familiarização com o problema, sendo considerado um passo inicial e fundamental para todo tipo de pesquisa.

Em vista disso, este artigo foi desenvolvido buscando descrever os conceitos e princípios que definem a Tecnologia Assistiva e a Simulação Virtual a partir de um estudo de revisão sistemática descritiva, desenvolvido com produção científica indexada nas seguintes bases eletrônicas de dados: BDENF, LILACS, MEDLINE, SCIELO e SCIENCEDIRECT, que enfocam a Tecnologia Assistiva ou Assistive Thecnology como descritor nuclear e Simulação Virtual ou Virtual Simulation como descritor complementar.

A revisão sistemática proporciona uma síntese do conhecimento baseado em pesquisas, relativo a um tópico específico (CILISKA; CULLUM; MARKS, 2001). Outros estudiosos consideram a revisão sistemática como um recurso para guiar a prática profissional e identificar a necessidade de futuras pesquisas (BANNINGAN; DROOGAN; ENTWISTLE, 1997, p. 52). Sendo assim, o recorte temporal abrangeu o período compreendido entre janeiro de 2009 a junho de 2014.

O estudo também foi construído por meio de levantamento de dados encontrados na literatura existente e pesquisa bibliográfica sobre a Tecnologia Assistiva e a Simulação Virtual, visando a busca e o entendimento dos conceitos de ambas as temáticas e suas aplicações no design.

Após o levantamento de dados, os artigos foram divididos por área de conhecimento e frequência de aparecimento em cada uma delas. Utilizou-se a classificação por área temática, para o tratamento dos dados, com o objetivo de se obter um panorama sobre pesquisas desenvolvidas na grande área (Ciências Sociais Aplicadas) e enfocando a Tecnologia Assistiva e a Simulação Virtual em suas várias aplicações e definições nas ciências e, em específico, no Design.

\section{FUNDAMENTACÃO TEÓRICA}

O presente artigo aborda as relações entre o design, a Tecnologia Assistiva e a Simulação Virtual, fazendo com que os futuros profissionais da área de design tenham um olhar que se dedica à investigação dos processos e ao desenvolvimento de produtos voltados a pessoas que apresentam dificuldades na linguagem, movimentos, interação (paralisia cerebral, autismo, deficiência 
múltipla, entre outros).

Nessa perspectiva, o design visa solucionar transdisciplinarmente algum problema ou alguma necessidade, pois já há exemplos na medicina de softwares e cirurgias que poderão ser feitas a partir de simulação 3D. E dessa maneira, o design torna-se parte ativa nos processos de transformação.

Para entender melhor a relação entre a Tecnologia Assistiva e a Simulação Virtual, primeiramente cabe destacar os conceitos que definem cada área.

\subsection{TECNOLOGIA ASSISTIVA}

Com frequência, o termo Tecnologia Assistiva tem aparecido no dia-a-dia dos usuários, mas nem todos sabem o seu significado. Muitas vezes, as pessoas o utilizam para denominar um conjunto de equipamentos específicos voltados para atender as necessidades das pessoas com deficiência, por isso a utilização errônea do termo no plural Tecnologias Assistivas . Em vista disso, por ser um termo ainda novo, é utilizado para identificar todo o arsenal de Recursos e Serviços que contribuem para proporcionar ou ampliar habilidades funcionais de pessoas com deficiência e consequentemente promover Vida Independente e Inclusão. (SARTORETTO; BERSCH, 2014).

A pessoa com deficiência segundo a Secretaria de Assistência Social/ Ministério da Previdência e Assistência Social SAS/MPAS (BRASIL, 1996, p. 7) é considerada como "[...] aquela que apresenta em caráter permanente, perdas ou anormalidades em sua estrutura ou função psicológica, fisiológica ou anatômica, decorrentes de causas congênitas e/ou adquiridas.

Possivelmente esta incompatibilidade de conceitos possa ser gerada por possuir a palavra tecnologia junto ao termo, fazendo com o que a Tecnologia Assistiva seja interpretada como algo que só esteja aliada a produtos tecnológicos, mas deve-se utilizar da tecnologia para justamente resolver problemas de ordem social e atender as necessidades diárias não somente das pessoas com deficiência. Assim como menciona Radabaugh (1993 apud BERSCH, 2013, p. 2) Para as pessoas sem deficiência a tecnologia torna as coisas mais fáceis. Para as pessoas com deficiência, a tecnologia torna as coisas possíveis. Sendo assim, a TA ${ }^{1}$ deve ser entendida como um auxílio que promoverá a ampliação de uma habilidade funcional deficitária ou possibilitará a realização da função desejada e que se encontra impedida por circunstância de deficiência ou pelo envelhecimento. (BERSCH, 2013, p. 2). O que torna evidente que a Tecnologia Assistiva não atende somente as pessoas com deficiência, mas também idosos que necessitam de auxílio para realizar atividades que antes faziam parte da sua 
rotina diária e que agora se tornam dificultosas.

Ainda sobre a definição do termo Tecnologia Assistiva no Brasil, o que influenciou foi a tradução de termos já utilizados em outros países, como Ajudas Técnicas. De acordo com o Secretariado Nacional para a Reabilitação e Integração das Pessoas com Deficiência (SNRIPD) de Portugal:

\begin{abstract}
Entende-se por ajudas técnicas qualquer produto, instrumento, estratégia, serviço e prática utilizada por pessoas com deficiência e pessoas idosas, especialmente, produzido ou geralmente disponível para prevenir, compensar, aliviar ou neutralizar uma deficiência, incapacidade ou desvantagem e melhorar a autonomia e a qualidade de vida dos indivíduos (PORTUGAL, 2007 apud BERSCH, 2013, p. 3).
\end{abstract}

E o conceito elaborado e proposto por uma comissão de países da União Europeia no documento "Empowering Users Through Assistive Technology" EUSTAT, afirma que,

[...] em primeiro lugar, o termo tecnologia não indica apenas objetos físicos, como dispositivos ou equipamento, mas antes se refere mais genericamente a produtos, contextos organizacionais ou modos de agir, que encerram uma série de princípios e componentes técnicos (EUROPEAN COMMISSION, 1998).

Em vista disso, no Brasil o Comitê de Ajudas Técnicas - CAT, instituído pela PORTARIA No 142, DE 16 DE NOVEMBRO DE 2006 propõe o seguinte conceito para a Tecnologia Assistiva:

Tecnologia Assistiva é uma área do conhecimento, de característica interdisciplinar, que engloba produtos, recursos, metodologias, estratégias, práticas e serviços que objetivam promover a funcionalidade, relacionada à atividade e participação de pessoas com deficiência, incapacidades ou mobilidade reduzida, visando sua autonomia, independência, qualidade de vida e inclusão social. (ATA VII - Comitê de Ajudas Técnicas (CAT) - Coordenadoria Nacional para Integração da Pessoa Portadora de Deficiência (CORDE) - Secretaria Especial dos Direitos Humanos - Presidência da República) (BRASIL, 2006b). 
Assim percebe-se o esclarecimento sobre a definição do termo Tecnologia Assistiva, que engloba diversas práticas que visam atender da melhor maneira possível às pessoas com deficiência para que elas executem suas tarefas diárias como qualquer outra pessoa, até mesmo crianças que estão em idade escolar podem ser auxiliadas por meio da TA,

[...] as ajudas técnicas e a tecnologia assistiva constituem um campo de ação da educação especial que têm por finalidade atender o que é específico dos alunos com necessidades educacionais especiais, buscando recursos e estratégias que favoreçam seu processo de aprendizagem, habilitando-os funcionalmente na realização das tarefas escolares (BRASIL, 2006a, p. 19).

Além dos mais, sabe-se que a deficiência é considerada historicamente como uma condição desvantajosa perante a sociedade, porém só se torna uma condição de desequilíbrio a partir de comparações que são geralmente feitas assim como mencionam Violante e Leite (2011, p. 78) "[...] aquela pessoa, com alguma deficiência, em relação aos demais membros do seu grupo, ou seja, a ideia de desvantagem só se faz pertinente quando se considera e julga determinado indivíduo em relação aos outros dentro de um grupo específico".

Segundo Mello (2010, p. 68) "A deficiência afeta, no mundo inteiro, pelo menos 600 milhões de pessoas, das quais 400 milhões vivem nos países em vias de desenvolvimento. Pode-se estimar que há um total de 79 milhões de pessoas com deficiência na América Latina e Caribe. E segundo pesquisa realizada pelo Centro de Pesquisas Sociais da Fundação Getúlio Vargas nos últimos 5 anos, existem hoje no Brasil, aproximadamente 27 milhões de pessoas com deficiência. Esse número equivale a $14,5 \%$ de toda a população brasileira. Além disso, cerca de 10 mil pessoas por mês, adquirem algum tipo de deficiência, seja por acidentes de trânsito ou por armas de fogo. Assim como afirma Pereira et al. (2013, p. 94) A população de pessoas deficientes vem crescendo a cada dia, gerando problemáticas sociais que interferem diretamente na qualidade de vida deste segmento .

Em vista disso, o governo elabora leis e decretos visando a integração e reintegração social desses indivíduos que encontram diversas dificuldades para ter acesso à educação, à mobilidade e, principalmente, ao trabalho. O Decreto no. 3.298 de 20/12/1999 (BRASIL, 1999) regulamenta a Lei no 7.853, de 24 de outubro de 1989 que dispõe sobre a Política Nacional para a Integração da Pessoa Portadora de Deficiência, consolidando as normas de proteção, dentre elas estão as que se referem à habilitação e reabilitação profissional, bem como ao acesso ao trabalho que devem estar em consonância com as necessidades e limitações tecnológicas e operacionais da empresa que emprega as pessoas 
com deficiência. O Decreto nํ. 3.298 de 99 considera a pessoa com deficiência a que se enquadra nas seguintes categorias: deficiência física, deficiência auditiva, deficiência visual e deficiência intelectual.

\title{
A Deficiência física é considerada uma
}

\begin{abstract}
Alteração completa ou parcial de um ou mais segmentos do corpo humano, acarretando o comprometimento da função física, apresentando-se sob a forma de paraplegia, paraparesia, monoplegia, monoparesia, tetraplegia, tetraparesia, triplegia, triparesia, hemiplegia, hemiparesia, ostomia, amputação ou ausência de membro, paralisia cerebral, nanismo, membros com deformidade congênita ou adquirida, exceto as deformidades estéticas e as que não produzam dificuldades para o desempenho de funções (BRASIL, 1999).
\end{abstract}

A Deficiência auditiva conforme o Decreto, é considerada a perda bilateral, parcial ou total, de quarenta e um decibéis $(\mathrm{dB})$ ou mais, aferida por audiograma nas frequências de $500 \mathrm{HZ}, 1.000 \mathrm{HZ}, 2.000 \mathrm{~Hz}$ e $3.000 \mathrm{~Hz}$.

\section{A Deficiência visual está relacionada com a}

Cegueira, na qual a acuidade visual é igual ou menor que 0,05 no melhor olho, com a melhor correção óptica; a baixa visão, que significa acuidade visual entre 0,3 e 0,05 no melhor olho, com a melhor correção óptica; ou a ocorrência simultânea de quaisquer das condições anteriores (BRASIL, 1999).

Já a Deficiência intelectual se refere ao

Funcionamento intelectual significativamente inferior à média, com manifestação antes dos dezoito anos e limitações associadas a duas ou mais áreas de habilidades adaptativas, tais como:

a.comunicação;

b.cuidado pessoal;

c. habilidades sociais;

d.utilização dos recursos da comunidade; 


\author{
e.saúde e segurança; \\ f. habilidades acadêmicas; \\ g.lazer; \\ h.trabalho (BRASIL, 1999).
}

A partir de todas as questões apresentadas, percebe-se a importância dos profissionais de design e também dos diversos profissionais de outras áreas, como a Arquitetura, a Engenharia, a Fisioterapia etc., estarem envolvidos multidisciplinarmente e em constante atualização para projetar soluções visando o auxílio para a vida diária; CAA (CSA) Comunicação aumentativa (suplementar) e alternativa; Recursos de acessibilidade ao computador; Sistemas de controle de ambiente; Projetos arquitetônicos para acessibilidade; Órteses e próteses; Adequação Postural; Auxílios de mobilidade; Auxílios para cegos ou com visão subnormal; Auxílios para surdos ou com déficit auditivo; Adaptações em veículos; todas categorias de Tecnologia Assistiva classificadas com base nas diretrizes gerais da ADA (American with Disabilities Act). Essas classificações são importantes, pois servirá para a pesquisa e o desenvolvimento de produtos visando a identificação de recursos disponíveis e mais apropriados ao atendimento da necessidade do indivíduo em específico. Qualquer que seja o motivo para projetar um produto, busca-se atender às necessidades humanas, sejam elas novas ou não. O desenvolvimento de projetos de produtos é uma atividade multidisciplinar, além disso, trata-se de um processo que requer grande quantidade de informação, conhecimento científico e técnico, por parte da equipe responsável, bem como, de uma grande capacidade criativa para poder responder às necessidades dos usuários.

O principal desafio do design na contemporaneidade é, justamente, desenvolver e/ou suportar o desenvolvimento de soluções a questões de alta complexidade, que exigem uma visão alargada do projeto, envolvendo produtos, serviços e comunicação, de forma conjunta e sustentável. (KRUCKEN, 2009, p. 23).

Portanto, a "[...] tecnologia assistiva deve ser compreendida como resolução de problemas funcionais, em uma perspectiva de desenvolvimento das potencialidades humanas, valorização de desejos, habilidades, expectativas positivas de aprendizagem e qualidade de vida". (MELLO, 2010, p. 73).

\title{
3.2 Simulação Virtual
}

Aliado à Tecnologia Assistiva, o objetivo desse artigo é poder realizar também, um levantamento de dados e revisão bibliográfica sobre a Simulação 
Virtual, pois justamente esta servirá de ferramenta ou meio para possivelmente se desenvolver produtos, ambientes virtuais, ou ainda postos de trabalho que possam auxiliar as pessoas com deficiência em suas tarefas diárias.

Para Ziolek e Kruithof (2000), o processo de modelagem e simulação humana pode ser dividido em três grandes áreas: o ambiente (incluindo desenhos em CAD computer aided design projeto auxiliado por computador), os manequins (ou modelos digitais humanos construídos com dados antropométricos) e as análises (possíveis a partir da simulação).

A simulação tanto humana quanto de produtos traz muitos benefícios para diversas áreas e tem conquistado espaços em grandes empresas e instituições. Estes benefícios são segundo Braatz et al. (2012, p. 82) [...] ganho de tempo no processo de projeto, auxílio na comunicação entre os diferentes atores do projeto e entre projetistas e usuários, e a antecipação das considerações que envolvem a possível atividade futura de trabalho .

Para realizar essas tarefas e gerar todos esses benefícios devem ser utilizados softwares de modelagem 3D, para simulação de movimentos dos usuários, de imersão em um ambiente virtual no qual a pessoa com deficiência possasimular situações da sua vida diária, realizar simples tarefas, principalmente devido aos seus movimentos que, na maioria das vezes, são limitados, [...] tais habilidades se traduzem em competências, compreendidas como uma capacidade do tipo particular, adquirida mediante o esforço pessoal. (TEIXEIRA, 2003, p. 7-8). Para isso seriam utilizados os programas AutoCAD, 3DStudio e a linguagem VRML -Virtual Reality Modeling Language-, ou Linguagem de Modelamento da Realidade Virtual (AMES; NADEAU; MORELAND, 1996; HARTMAN; WERNECKE, 1996), que é um dos formatos mais utilizados e alia qualidade visual com velocidade de navegação e fácil disponibilização através da Internet (AYMONE, 2003, p. 2).

A programação em linguagem VRML é feita através de qualquer editor de texto e exige a investigação dos seus comandos. É uma linguagem bastante ampla que permite o modelamento de objetos [...] complexos por faces triangulares e quadriláteras, [...]. (AYMONE, 2003, p. 6). Existem plugins gratuitos para o Internet Explorer que possibilitam passeios em realidade virtual pelos ambientes de forma interativa e em tempo real, utilizando os botões que permitem diferentes movimentações de câmeras, facilitando a exploração do ambiente virtual. (AYMONE, 2003, p. 10). Os plug-ins podem reproduzir fielmente o espaço real e possibilitar a interatividade com o usuário. Os procedimentos utilizados para a otimização são acessíveis aos usuários dos programas AutoCAD e 3DStudio, mas podem também estar disponíveis e serem empregados em outros programas. (AYMONE, 2003, p. 10). A otimização dos modelos agiliza consideravelmente a navegação em tempo real sem perda 
de qualidade gráfica, não sendo necessários computadores potentes para se atingir uma boa velocidade de navegação. Os procedimentos utilizados para a otimização são acessíveis aos usuários dos programas AutoCAD e 3DStudio.

Além de todas essas possibilidades de utilização da simulação virtual, percebe-se que possivelmente, esta ferramenta possa ser utilizada para a modelagem de produtos e teste de usabilidade, servindo para áreas que estão envolvidas com processo de projeto de produto. Segundo Daniellou (2002, p. 75) "A aplicação de simulação no processo de projeto de engenharia, durante um estudo ergonômico, deve ter fundamentação conceitual e metodológica para obter resultados efetivos".

Todavia, é conveniente ressaltar dois problemas acerca deste assunto: a) que no Brasil, assim como em muito outros países, há uma carência muito grande de pesquisas antropométricas que ultrapassem os limites de levantamentos com populações específicas espacialmente localizadas; e b) apesar da existência de equipamentos de grande precisão e rapidez para aquisição de dados antropométricos (sobretudo scanner 3D), os principais softwares comerciais ainda não estão aptos ao uso desta rica fonte de dados. (BRAATZ et al., 2012, p. 82), por isso essa pesquisa torna-se relevante para auxiliar no desenvolvimento de trabalhos futuros na área do Design.

\section{RESULTADOS}

$\mathrm{Na}$ busca realizada em todas as bases de dados já citadas, foram encontrados 291 artigos relacionados aos descritores Tecnologia Assistiva ou Assistive Thecnology e Simulação Virtual ou Virtual Simulation.

Na base de dados Medline, foram encontrados 26 artigos, utilizando a seguinte descrição durante a pesquisa Assistive AND Technology AND Virtual AND Simulation.

$\mathrm{Na}$ base de dados LILACS, não foram encontrados artigos utilizando como descritores Tecnologia Assistiva ou Assistive Thecnology e Simulação Virtual ou Virtual Simulation. Esclarece-se que também não foram encontrados artigos nas bases de dados BDENF e SCIELO. Já na SCIENCEDIRECT foram encontrados 265 artigos em revistas. Destes foram encontrados maior número de publicações no período de 2014, 65 artigos.

A seguir, quadro que detalha a frequência dos artigos por área do conhecimento: 
Quadro 1 - Artigos por área de conhecimento

\begin{tabular}{|l|l|l|l|}
\hline BASE DE DADOS & TOTAL & ÁREAS & FREQUÊNCIA \\
\hline MEDLINE & 26 & Ciência da Computação & 6 \\
\hline & & Engenharia & 8 \\
\hline & & Psicologia & 1 \\
\hline LILACS & Medicina & 9 \\
\hline BDENF & 0 & - & - \\
\hline SCIELO & 0 & - & - \\
\hline SCIENCEDIRECT & 0 & - & - \\
\hline & 265 & - & - \\
\hline & & Ciência da Computação & 72 \\
\hline & & Engenharia & 68 \\
\hline & & Pedicologia & 25 \\
\hline & & Ciências Sociais Aplicadas & 8 \\
\hline
\end{tabular}

Fonte: Do autor.

\section{DISCUSSÃO}

O resultado do estudo evidenciou que a abordagem da Tecnologia Assistiva e da Simulação Virtual se inicia a partir de 1990. Das publicações americanas e inglesas encontradas na base de dados Medline, ressalta-se a maior concentração da temática na área da Medicina, seguida da Engenharia.

Verificou-se que nas publicações concentradas nas bases de dados LILACS, BDENF e SCIELO a abordagem sobre Tecnologia Assistiva e Simulação Virtual é escassa, podendo-se dizer nula, pois não foi encontrado nenhum artigo durante a pesquisa utilizando os descritores já citados. Já na base de dados SCIENCEDIRECT, a área de conhecimento que mais trata desta temática é a Ciência da Computação, seguida da Engenharia e da Medicina.

Constatou-se que a Tecnologia Assistiva e a Simulação Virtual como objetos de estudo integram a realidade das pesquisas internacionais, contabilizando 291 artigos. Porém, a representatividade desta abordagem na área de Ciências Sociais Aplicadas ainda apresenta pouca expressão, evidenciando-se em apenas 8 artigos, o que representa 2,74\% do total dos estudos encontrados.

Dos 269 estudos analisados disponíveis nas bases de dados pesquisadas, a maioria deles utilizava a abordagem quantitativa na aproximação ao conceito, para isso utilizaram como instrumentos de coleta de dados questionários e entrevistas. 
Os artigos consultados apontam controvérsias quanto aos conceitos de Tecnologia Assistiva. "Assistive Technologies include a large number of products, systems and services which aim to compensate for a loss of autonomy, in the medical and social domains." (NEWELL, 2003). Assim como já foi discutido neste artigo anteriormente, o termo Tecnologia Assistiva não deve ser utilizado no plural e que já está agregado ao conceito que este também se refere aos produtos tecnológicos que atendem somente às pessoas com deficiência. "Assistive Technologies aim to provide a support to disabled people in their everyday life and for their social participation." (PLOS et al., 2012, p. 534). Sendo assim, há certa incoerência entre as publicações internacionais e as nacionais referente à definição do termo Tecnologia Assistiva. Já referente à Simulação Virtual a principal característica das pesquisas publicadas se referem à exploração do ambiente virtual e aprendizado, por parte dos participantes, bem como, aos recursos de transferência que garantem que as habilidades desenvolvidas no ambiente virtual são úteis na resolução de problemas no mundo real.

Entre as revistas onde é publicada a maioria dos trabalhos sobre essa temática, destaca-se a Computers \& Education, com o tema que mais se destaca que é estudante.

Além disso, a maioria dos estudos discute a Tecnologia Assistiva e a Simulação Virtual utilizando cenários animados em 3D em ambientes virtuais de aprendizagem para ajudar as pessoas com deficiência.

\section{CONCLUSÃO}

Durante a elaboração deste artigo, percebeu-se que há uma lacuna nas publicações que utilizam os conceitos Tecnologia Assistiva e Simulação Virtual na América Latina, fato que contribui para a relevância deste estudo.

Desta forma, acredita-se que, dado seu potencial, a Simulação Virtual é um conceito que pode ser significativo para o desenvolvimento de pesquisas e de produtos de Tecnologia Assistiva, contribuindo para reflexões na área do design.

Além disso, torna-se de suma importância a instrumentalização dos designers para a capacitação e formação de equipes, a fim de que possam identificar problemas de design em produtos voltados para a Tecnologia Assistiva, de modo a colaborar em equipes multidisciplinares das quais estejam constituídas por profissionais que lidam com ferramentas e programas de Simulação Virtual. O enfoque da Simulação Virtual pode ser uma fonte de inspiração e de orientação da nossa atenção e da nossa ação. Porém, compete 
a cada designer definir o que pretende e o que pode fazer com este instrumento de trabalho, pois a Simulação Virtual se apresenta como uma ferramenta computacional, uma visão da vida que possibilita uma nova interpretação dos fatos do dia-a-dia, principalmente para pessoas com deficiência.

Em vista disso, o designer deve auxiliar na implementação de soluções mais eficientes e orientadas para as pessoas com deficiência no contexto social, adaptando conhecimentos técnicos e científicos, até mesmo de outras áreas, a uma linguagem e realidade que possibilitem o entendimento real das situações.

O conhecimento sistemático sobre a Tecnologia Assistiva e a Simulacão Virtual é instrumento válido para todos os designers e pesquisadores da área que pretendem aperfeiçoar seu campo profissional.

Assim, é responsabilidade da sociedade em prover condições estruturais, sociais e econômicas para que todos os sujeitos exerçam seus direitos e obrigações de cidadãos, de modo que essa mesma sociedade deva prover os ajustes necessários no suprimento às demandas de todo e qualquer indivíduo (SASSAKI, 2006).

\section{REFERÊNCIAS}

AMES, A. L.; NADEAU, D. R.; MORELAND, J. L. The VRML Sourcebook. New York: John Wiley \& Sons, 1996.

AYMONE, J. L. F. A otimização de modelos em realidade virtual para engenharia. In: COBENGE, 31., Rio de Janeiro. Anais... Rio de Janeiro: Cobenge, 2003.

BANNINGAN; DROOGAN; ENTWISTLE, 1997

BERSCH, R. Introdução à tecnologia assistiva. Porto Alegre: Assistiva Tecnologia E Educação, 2013.

BRAATZ, D. et al. Simulação humana digital na concepção de postos de trabalho: estudo comparativo de casos. Gestão \& Produção, São Carlos, v. 19, n. 1, p. 79-92, 2012.

BRASIL. Decreto no. 3.298, de 20 de dezembro de 1999. Regulamenta a Lei no 7.853, de 24 de outubro de 1989, dispõe sobre a Política Nacional para a Integração da Pessoa Portadora de Deficiência, consolida as normas de proteção, e dá outras providências. Disponível em: <http://www.planalto.gov.br/ccivil_03/decreto/d3298.htm>. 
Acesso em: 10 abr. 2014.

BRASIL. Ministério da Educação e Assistência Social. Secretaria de Assistência Social. A atenção à pessoa portadora de deficiência na área da assistência social. Brasília: 1996.

BRASIL: Ministério da Educação. Secretaria de Educação Especial. Sala de recursos multifuncionais: espaços para o atendimento educacional especializado. Brasília: MEC/SEESP, 2006a.

BRASIL. Secretaria de Direitos Humanos. Portaria no 163 de 29 de dezembro de 2006. Torna público o Regimento Interno do Comitê de Ajudas Técnicas. 2006b. Disponível em: <https://www.legisweb.com. br/legislacao/?id=196373>. Acesso em: 12 maio 2014.

CILISKA, D.; CULLUM, N.; MARKS, S. Evaluation of systematic reviews of treatment or prevention interventions. Evidence-Based Nursing, England, v. 4, n. 4, p. 100-4, Oct. 2001.

COOK, A. M.; HUSSEY, S. M. Assistive technologies: principles and practices. St. Louis: Mosby - Year Book, 1995.

DANIELLOU, F. A análise da atividade futura e a concepção de instalações externas. In: DUARTE, F. (Org.). Ergonomia e projeto na indústria de processo contínuo. Rio de Janeiro, 2002. p. 75-83.

\section{EUROPEAN COMMISSION. Empowering users trought assistive} technology. 1998. Disponível em: <http://www.siva.it/research/eustat/ index.html>. Acesso em: 5 maio 2014.

HARTMAN, J.; WERNECKE, J. The VRML 2.0 Handbook. New York: Silicon Graphics, 1996.

KRUCKEN, L. Design e território: valorização de identidades e produtos locais. São Paulo: Studio Nobel, 2009.

MELLO, A. G. Políticas públicas de educação inclusiva: oferta de tecnologia assistiva para estudantes com deficiência. Revista Habitus: Revista Eletrônica dos Alunos de Graduação em Ciências Sociais, Rio de Janeiro, v. 8, n. 1, p. 68-92, jul. 2010. Disponível em: <www.habitus.ifcs. ufrj.br>. Acesso em: 26 jul. 2010.

NEWELL, A. Inclusive design or assistive technology. In: CLARKSON, J. et al. (Ed.). Inclusive design: design for the whole population. United 
States: Springer, 2003. p. 172-181.

Pereira et al. (2013

PLOS, O. et al. A universalist strategy for the design of Assistive Technology. International Journal of Industrial Ergonomics, Amsterdam, v. 42, p. 533-541., 2012. Disponível em: <http://ac.els-cdn.com/S0169814112000881/1-s2.0S0169814112000881-main.pdf?_tid=ca118df2-004f-11e48ea5-00000aab0f01\&acdnat=1404130522_682acf505 8146bd737449981fc616b44>. Acesso em: 1 jun. 2014.

PRODANOV, C. C.; FREITAS, E. C. de. Metodologia e técnicas da pesquisa e do trabalho acadêmico. 2. ed. Novo Hamburgo: Feevale, 2013.

Sartoretto e Bersch (2014)

SASSAKI, R. K. Inclusão: construindo uma sociedade para todos. Rio de Janeiro: WVA, 2006.

TEIXEIRA, 2003

TRIVIÑOS, A. N. S. Introdução à pesquisa em ciências sociais: a pesquisa qualitativa em educação. São Paulo: Atlas, 2006.

VIOLANTE, R. R.; LEITE, L. P. A empregabilidade das pessoas com deficiência: uma análise da inclusão social no mercado de trabalho do município de Bauru, SP. Cadernos de Psicologia Social do Trabalho, São Paulo, v. 14, n. 1, jun. 2011.

ZIOLEK, S. A.; KRUITHOF, P. C. J. Human modeling \& simulation: a primer for practitioners. In: HFES, 44., 2000, San Diego. Proceedings... San Diego: HFES, 2000. 


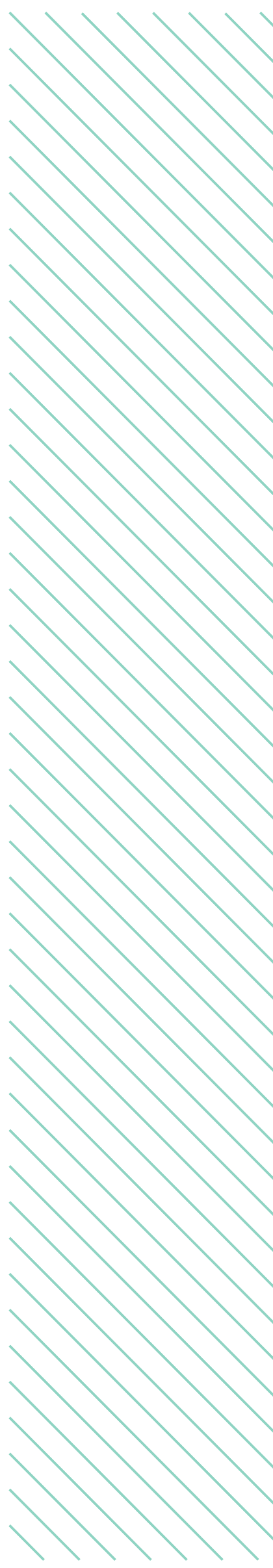

